\title{
CONTEOS CROMOSOMICOS EN PHILESIA MAGELLANICA J.F.GMEL. (LILIALES: PHILESIACEAE)
}

\section{CHROMOSOME COUNTS IN PHILESIA MAGELLANICA J.F.GMEL. (LILIALES: PHILESIACEAE)}

\author{
Pedro Jara-Seguel \& Andrés Fuentes-Ramírez \\ Escuela de Ciencias Ambientales, Facultad de Recursos Naturales, Universidad Católica de Temuco, \\ Casilla 15-D, Temuco-Chile. pjara@uct.cl
}

\begin{abstract}
Mitotic chromosome counts documented here for Philesia magellanica J.F.Gmel. (Philesiaceae) showed a diploid number $2 \mathrm{n}=30(\mathrm{x}=15)$, which is different to the numbers reported previously for the species. The $2 \mathrm{n}$ number of P. magellanica is similar to that documented previously for Lapageria rosea, which indicate a constancy of chromosome number within the family.
\end{abstract}

Philesia Comm. ex Juss. (Philesiaceae), con su única especie $P$. magellanica J.F.Gmel., se distribuye en Chile desde la provincia de Valdivia $\left(39^{\circ} \mathrm{S}\right)$ hasta Magallanes (50S) (Hoffmann 1997), mientras que en Argentina cubre también parte importante de la zona patagónica (Arroyo-Leuenberger 2001). Para este género, los antecedentes citogenéticos disponibles son confusos, puesto que distintos números cromosómicos han sido documentados. Así, en un estudio preliminar realizado en granos de polen en división de un espécimen, Cave (1966) describió un número haploide $\mathrm{n}=19$, mientras que Moore (1968), en un estudio florístico, documentó un número somático $2 \mathrm{n}=12$. Esos dos números cromosómicos descritos para Philesia han sido citados recurrentemente como caracteres diagnósticos en varias revisiones taxonómicas y listados florísticos que han incluido a Philesiaceae (Watson \& Dallwitz 1992, Arroyo-Leuenberger 2001, MBG 2006). Sin embargo, no existen registros de nuevos conteos de cromosomas mitóticos o meióticos que permitan corroborar la validez de esos números para el género.

Con el objeto de clarificar las confusiones existentes respecto del número cromosómico de $P$. magellanica, en el presente trabajo se informa el resultado de nuevos conteos de cromosomas mitóticos realizados en individuos de una población natural.

Se recolectaron plantas de una población de $P$. magellanica localizada en Chile, Provincia de Osorno, al sur del camino entre La Unión y Hueicolla, $875 \mathrm{~m}\left(40^{\circ} 11^{\prime} \mathrm{S} ; 7^{\circ} 26^{\prime} \mathrm{W}\right), 13-04-2005$, A. FuentesRamírez AF0405. Un ejemplar fue depositado en el Herbario no oficial (UCT) de la Escuela de Ciencias Ambientales, Universidad Católica de Temuco. Raíces de 5,0 mm de longitud fueron extraídas desde los rizomas de cinco plantas y se trataron con 8 Hidroxiquinolina $2 \mathrm{mM}$ a $8^{\circ} \mathrm{C}$ por $6 \mathrm{~h}$. Luego, fueron fijadas en una solución de etanol-ácido acético glacial $(3: 1 \mathrm{v} / \mathrm{v})$ a $4^{\circ} \mathrm{C}$ por $24 \mathrm{~h}$ y coloreadas con la reacción de Feulgen. Cromosomas mitóticos fueron obtenidos por aplastado de meristemas radiculares y fotografiados con una cámara digital OLYMPUS C-5050 conectada a un microscopio OLYMPUS CX31. Los cromosomas fueron contados sobre impresiones fotográficas ampliadas de veinte metafases.

Philesia magellanica presenta un complemento cromosómico diploide $2 \mathrm{n}=30(\mathrm{x}=15)$, en el cual se identifican tres pares de cromosomas acrocéntricos portadores de constricción secundaria y satélite en el brazo corto (Fig. 1). El número cromosómico descrito aquí para $P$. magellanica no concuerda con 
el número haploide $\mathrm{n}=19$ (Cave 1966) o con el número somático $2 \mathrm{n}=12$ (Moore 1968) documentados hace algunas décadas. Todos esos números cromosómicos podrían ser interpretados como polimorfismos poblacionales dentro del rango de distribución de la especie. Sin embargo, es factible también que los números $n=19$ y $2 n=12$ sean el resultado de conteos erróneos realizados en aquellos trabajos previos.

Una característica citogenética relevante que se ha observado dentro del orden Liliales es la constancia intra e interespecífica en los números cromosómicos básicos de aquellos géneros que han sido estudiados (i.e. Rhipogonum J.R.Forst. \& G.Forst. $\mathrm{x}=15$, Clintonia Raf. $\mathrm{x}=7$,Alstroemeria $\mathrm{L}$. $\mathrm{x}=8$, Bomarea Mirb. $\mathrm{x}=9$, Lilium L. $\mathrm{x}=12$, Schoenocaulon A.Gray $\mathrm{x}=8$, Toxicoscordion $\mathrm{Rydb}$. $\mathrm{x}=11$, Luzuriaga Ruiz \& Pav. $\mathrm{x}=10$, Leontochir Phil. $x=9$ ) (Beuzenberg \& Hair 1963, Li et al. 1996, Sanso \& Hunziker 1998, Marasek \& Orlikowska 2003, Zomlefer 2002, 2003, Jara-Seguel \& Zúñiga 2004,
Jara-Seguel et al. 2005, Palma-Rojas et al. 2007). Una excepción a esa constancia la constituye el género Smilax L., el cual presenta distintos números básicos a nivel interespecífico, con $\mathrm{x}=12,13,14,15$ y 16 (Speese 1939) y cuya variación podría estar relacionada con el amplio rango geográfico que cubren sus especies en ambos hemisferios. En el caso de Philesiaceae, el número cromosómico de la población de Philesia analizada en este trabajo es similar a aquel documentado previamente para especímenes de tres poblaciones de su género hermano Lapageria $(2 \mathrm{n}=30)$ (Titov de Tschicshow 1954, Hanson et al. 2003, Jara-Seguel \& Zúñiga 2004), lo cual sugiere constancia del número básico $\mathrm{x}=15$ dentro de la familia. Adicionalmente, el número básico $\mathrm{x}=15$ es un carácter que está presente también en los géneros gondwánicosRhipogonum (Rhipogonaceae) (Beuzenberg \& Hair 1963) y Smilax (Smilacaceae) (Speese 1939), ambos filogenéticamente muy cercanos a Philesiaceae (Vinnersten \& Bremer 2001).

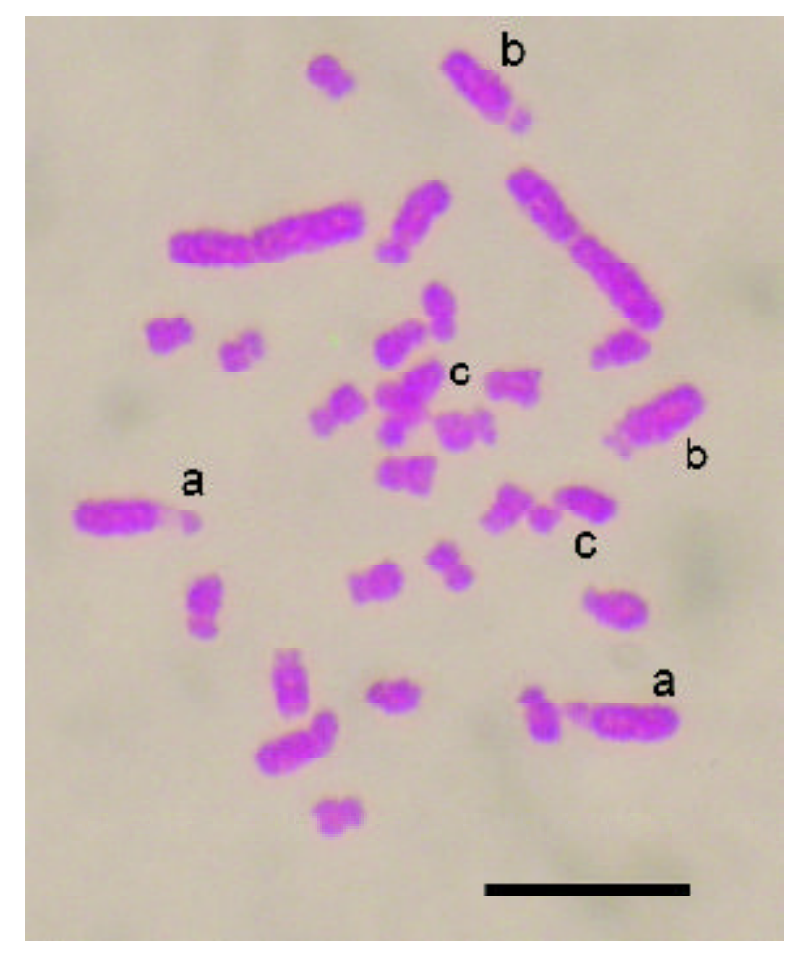

FIGURA 1 . Metafase mitótica de Philesia magellanica $2 \mathrm{n}=30$. a, b y c identifican pares de cromosomas acrocéntricos con constricción secundaria y satélite en el brazo corto. Barra $=10 \mu \mathrm{m}$.

Figure 1. Mitotic metaphase of Philesia magellanica $2 \mathrm{n}=30$. a, $\mathrm{b}$ and c identify achrocentric chromosome pairs with secondary constriction and satellite on the short arm. Bar $=10 \mu \mathrm{m}$. 
Estudios citogenéticos adicionales que incluyan un mayor número de poblaciones de Philesia y Lapageria (i.e. morfología de cariotipos, patrones de bandas C, tamaños genómicos, hibridación in situ, apareamiento meiótico) permitirían obtener mayores detalles acerca de la organización del genoma de ambos géneros monotípicos, reforzando así el escaso conocimiento existente sobre relaciones evolutivas dentro de la familia. No obstante, los datos citogenéticos descritos y discutidos en el presente trabajo, aunque parciales, respaldan su clasificación como los dos únicos géneros adscritos a Philesiaceae, tal como ha sido clarificado previamente por ArroyoLeuenberger (2001) basado en los antecedentes morfológicos y moleculares disponibles en la literatura.

\section{AGRADECIMIENTOS}

A Enrique Hauenstein por la lectura del manuscrito y a Alicia Marticorena por el envío de literatura referente al estudio de gametofitos en Philesia. Financiado parcialmente por Proyecto DGIUCT 2005-4-02.

\section{BIBLIOGRAFIA}

Arroyo-Leuenberger, S. 2001. Flora Fanerogámica Argentina: Philesiaceae, Asteliaceae y Luzuriagaceae. Pro-Flora CONICET 72: 1-7.

Beuzenberg, E. \& B. Hair. 1963. Contributions to a chromosome atlas of the New Zealand flora -5 , miscellaneous families. New Zealand Journal of Botany 1: 53-67.

CAVE, M. 1966. The female gametophytes of Lapageria rosea and Philesia magellanica. Gayana Botánica 15: 25-31.

Hanson, L., R. Brown,A. Boyd, M. Johnson \& M. Bennett. 2003. First nuclear DNA C-values for 28 angiosperm genera. Annals of Botany 91: 31-38.

Hoffmann, A. 1997. Flora silvestre de Chile, Zona Araucana. Ediciones Fundación Claudio Gay, Santiago. 258 pp.
Jara-Seguel, P. \& C. ZúÑiga. 2004. El Cariotipo de Lapageria rosea Ruizet Pav.(Liliales: Philesiaceae). Gayana Botánica 61: 76-78.

Jara-Seguel, P. \& C. Z ÚñIga. 2005. Chromosome numbers in Chilean species of Luzuriaga R. et P. (Luzuriagaceae). Gayana Botánica 62: 53-55.

Jara-Seguel, P., C. Palma-Rojas \& E. Von Brand. 2005. Cbanding pattern in the geophytic Leontochirovalle $i$ (Alstroemeriaceae). Belgian Journal of Botany 138: 85-88.

Li, S., Z. Chang \& Y. Yuan. 1996. The origin and dispersal of the genus Clintonia Raf. (Liliaceae): evidence from its cytogeography and morphology. Caryologia 49: 125-135.

M arasek, A. \& T. Orlikowska. 2003. Karyology of nine Lily genotypes. Acta Biologica Cracoviensia, Series Botanica 45: 159-168.

MBG, 2006. Angiosperm Phylogeny Website, Missouri Botanical Garden, URL: http://www.mobot.org/ MOBOT/Research/APweb/welcome.html Viewed: May 22, 2006.

Moore, D. 1968. The vascular flora of the Falkland Islands. In: British Antarctic Survey, Scientific Report 60. London. 201 pp.

Palma-Rojas, C., P. Jara-Seguel \& E. Von Brand. 2007. Karyological studies in Chilean species of Bomarea and Leontochir (Alstroemeriaceae). New Zealand Journal of Botany 45: 299-303.

SANSO, M. y J. HunZiKer. 1998. Karyological studies in Alstroemeria and Bomarea (Alstroemeriaceae). Hereditas 129: 67-74.

SPEese, B. 1939. Mitosis in leaves of Smilax. American Journal of Botany 26: 852-855.

Titov de Tschicshow, N. 1954. Estudios citológicos en Lapageria rosea Ruiz et Pavón. Boletín de la Sociedad de Biología de Concepción. 29: 3-6.

Vinnersten, A. \& K. Bremer. 2001. Age and biogeography of major clades in Liliales. American Journal of Botany 88: 1695-1703.

WATSON, L. \& J. Dallwitz. 1992. The families of flowering plants, illustrations, identification, and information retrieval. URL: http://www.biodiversity.uno.edu/ delta/ Viewed: October 23, 2005.

Zomlefer, W. 2002. Documented chromosome numbers 2002: 1. Chromosome number of Stenanthium (Liliales: Melanthiaceae) and its significance in the taxonomy of tribe Melanthieae. Sida 20: 221-226.

ZomLEFER, W. 2003. Documented chromosome numbers 2003: 1. Chromosome number of Toxicoscodion nutallii (Liliales: Melathiaceae) and clarification of the genus. Sida 20: 1085-1092. 